Methods: We examined data on patients and their disease-related characteristics entered into the COVID-19 GRA provider registry from Ireland (24th March 2020 to 31st August 2020). Multivariable logistic regression was used to assess the association of demographic and clinical characteristics with hospitalisation.

Results: Of 105 patients, 47 (45.6\%) were hospitalised and 10 (9.5\%) died. Multivariable logistic regression analysis showed age $(\mathrm{OR}=1.06,95 \% \mathrm{Cl} 1.01$ to 1.10$)$, number of comorbidities (OR=1.93, $95 \% \mathrm{Cl} 1.11$ to 3.35), and glucocorticoid use $(\mathrm{OR}=15.01,95 \% \mathrm{Cl} 1.77$ to 127.16$)$ were significantly associated with hospitalisation. A diagnosis of inflammatory arthritis was associated with a lower odds of hospitalisation $(\mathrm{OR}=0.09,95 \% \mathrm{Cl} 0.02$ to 0.32$)$.

Conclusion: Increasing age, comorbidity burden, and glucocorticoid use were associated with hospitalisation, while a diagnosis of inflammatory arthritis was associated with lower odds of hospitalization.

Disclosure of Interests: Richard Conway Speakers bureau: Janssen, Roche Sanofi, Abbvie, Elena Nikiphorou Speakers bureau: AbbVie, Eli-Lilly, Gilead, Celltrion, Pfizer, Sanofi, Christiana Demetriou: None declared, Candice Low: None declared, Kelly Leamy: None declared, John Ryan: None declared, Ronan Kavanagh: None declared, Alexander Fraser: None declared, John Carey: None declared, Paul O'Connell: None declared, Rachael Flood: None declared, Ronan Mullan: None declared, David Kane: None declared, Philip Robinson Speakers bureau: UCB, Roche, Pfizer, Gilead, Janssen, Novartis, Eli Lilly, Abbvie, Grant/research support from: Abbvie, UCB, Novartis, Janssen, Pfizer, Jean Liew Grant/research support from: Pfizer, Rebecca Grainger Speakers bureau: Pfizer, Cornerstones, Janssen, Novartis, Abbvie, Geraldine McCarthy: None declared.

DOI: 10.1136/annrheumdis-2021-eular.420

\section{POS1163 CHARACTERISTICS AND OUTCOMES IN A REAL- WORLD COHORT OF RHEUMATOID ARTHRITIS PATIENTS WITH COVID-19}

$\underline{\mathrm{Y} . \mathrm{Ye}^{1}}, \mathrm{X} . \mathrm{Yue}^{1}$, W. Krueger ${ }^{1}$, L. Wegrzyn ${ }^{1} .{ }^{1}$ AbbVie Inc., Global Epidemiology, North Chicago, United States of America

Background: While some risk factors for severe COVID-19 outcomes have been identified for the general population and patients with rheumatic diseases (1-3), what drives these outcomes in specific rheumatic disease remains unclear. In addition, these findings need to be assessed across various observational data sources to ensure external validity.

Objectives: To describe the demographics, comorbidities, and severe COVID-19 outcomes among rheumatoid arthritis (RA) patients infected with SARS-CoV-2 in the United States.

Methods: A large nationwide electronic health record database (Optum, Inc.) in the United States, with data range between February 1, 2020 and September 17,2020 , was used to describe the demographics, comorbidities, and severe COVID-19 outcomes of RA patients with confirmed COVID-19 diagnosis (diagnosis for COVID-19 or positive PCR or antigen test). Patients with a single diagnosis of RA (ICD-10 code) before the diagnosis of COVID-19 were included. Patients missing age or sex, under 18 years of age on COVID-19 diagnosis date, or having less than 15 months of activity prior to COVID-19 diagnosis in the data source were excluded. We described demographics, comorbidities, and severe COVID-19 outcomes, including death, hospitalization, ICU admission, and acute respiratory insufficiency (ARI) identified between 14 days prior to and 30 days after COVID-19 diagnosis. Mean and standard deviation (SD) was reported for continuous variables. For categorical variables, count $(\mathrm{N})$ and proportion was reported.

Results: We identified 2,948 patients diagnosed with RA and infected with SARS-CoV-2 (mean age \pm SD: 62 years $\pm 16,77 \%$ female, $68 \%$ white). Of all identified patients, $38 \%$ were current or former smokers. For the 2,614 patients with $\mathrm{BMI}$ recorded, $78 \%$ were overweight or obese (mean $\mathrm{BMI} \pm \mathrm{SD}: 31.2 \pm 8.3$ ). The mean Charlson comorbidity index (CCI) was 3.6 (SD 3.2), with $87 \%$ of the study cohort having one or more comorbid condition, including hypertension (55\%), type 2 diabetes (26\%), COPD $(20 \%)$, moderate to severe asthma (17\%), coronary artery disease (17\%), chronic kidney disease $(13 \%)$, and heart failure (13\%). Severe COVID-19 outcomes occurred in 618 (21\%) patients. Among all RA patients with COVID-19, 137 patients (4.6\%) experienced ARI, 484 patients (16.4\%) were hospitalized (including 174 (5.9\%) admitted to the ICU), and 155 patients (5.3\%) died

Conclusion: Underlying medical conditions that are known or possible risk factors of severe illness from SARS-CoV-2 infection in the general population are common in this RA cohort from a large national EHR database. However, whether patients with RA are more vulnerable to severe COVID-19 outcome than the general population requires adjustment by age and other important confounders.

\section{REFERENCES:}

[1] Gianfrancesco M, Hyrich KL, Al-Adely S, Carmona L, Danila MI, Gossec L, et al. Characteristics associated with hospitalisation for COVID19 in people with rheumatic disease: data from the COVID-19 Globa Rheumatology Alliance physician-reported registry. Ann Rheum Dis. 2020;79(7):859-66.

[2] Williamson EJ, Walker AJ, Bhaskaran K, Bacon S, Bates C, Morton CE, et al. Factors associated with COVID-19-related death using OpenSAFELY Nature. 2020;584(7821):430-6.

[3] Gold JAW, Wong KK, Szablewski CM, Patel PR, Rossow J, da Silva J, et al. Characteristics and Clinical Outcomes of Adult Patients Hospitalized with COVID-19 - Georgia, March 2020. MMWR Morb Mortal Wkly Rep. 2020;69(18):545-50.

Disclosure of Interests: Yizhou Ye Shareholder of: AbbVie Inc. and Pfizer Inc. Employee of: AbbVie Inc., Xiaomeng Yue Employee of: AbbVie Inc., Whitney Krueger Shareholder of: AbbVie Inc., Employee of: AbbVie Inc., Lani Wegrzyn Shareholder of: AbbVie Inc., Employee of: AbbVie Inc.

DOI: 10.1136/annrheumdis-2021-eular.565

\section{\begin{tabular}{|l|l}
\hline POS1164 USE OF TELEMEDICINE FOR FOLLOW- \\
\hline
\end{tabular} UP OF SLE PATIENTS WITH NEPHRITIS IN THE COVID-19 OUTBREAK ("TELESLE"): THE 6-MONTH RESULTS OF A RANDOMIZED CONTROLLED TRIAL}

${ }^{\text {H. So }}{ }^{1}$, E. Chow ${ }^{1}$, T. K. LI ${ }^{1}$, S. L. Lau ${ }^{1}$, I. T. Cheng ${ }^{1}$, C. C. Szeto ${ }^{1}$, L. S. Tam ${ }^{1}$. ${ }^{1}$ The Chinese University of Hong Kong, Medicine and Therapeutics, Hong Kong, Hong Kong (SAR)

Background: Patients with lupus nephritis (LN) might be more susceptible to COVID-19 due to the underlying disease, co-morbidities and use of immunosuppressants. We hypothesized that telemedicine (TM) could be a well-accepted mode of health-care delivery minimizing the risk of exposure to the severe acute respiratory syndrome coronavirus 2 (SARS-CoV-2), while maintaining disease control in these patients.

Objectives: To evaluate the short-term patient satisfaction, compliance, disease control and infection risk of TM compared with standard in-person follow-up (FU) for patients with LN during COVID-19.

Methods: This was a single-center randomized-controlled study. Consecutive patients followed at the LN clinic were randomized to either TM (TM group) or standard FU (SF group) in a 1:1 ratio. Patients in the TM group received scheduled follow-ups via videoconferencing. SF group patients continued conventional in-person outpatient care. The 6-month data were compared.

Results: From June to December 2020, 122 patients were randomized (TM: 60, SF: 62) and had attended at least 2 FU visits. There were no baseline differences, including SLEDAI-2k and proportion of patients in lupus low disease activity state (LLDAS), between the 2 groups except a higher physician global assessment score (PGA) in the TM group (mean $0.67 \pm 0.69$ vs $0.45 \pm 0.60, p=0.003$ ) (Table 1). The mean FU duration was $19.8 \pm 4.5$ weeks. When comparing the most recent visit, the mean waiting time between enter ing the clinic waiting room (virtual or real) and seeing a rheumatologist (virtual or in-person) was significantly shorter in the TM group $(22.5 \pm 28.6$ vs $68.9 \pm 40.7$ minutes, $p<0.001$ ) (Figure $1 \mathrm{~A}$ ). The mean overall patient satisfaction score was higher in the TM group (mean $2.19 \pm 0.61$ vs $1.89 \pm 0.78$, $p=0.042$ ). The results of the post-consultation satisfaction questionnaire are shown in Figure 1B. The number of visits was similar in the two groups (TM: $3.1 \pm 1.3$ vs $S F: 3.0 \pm 1.2, p=0.981)$. However, there was a trend suggesting that alternative mode of $\mathrm{FU}$ was requested more frequently in the TM group than the SF group (TM: $12 / 60,20.0 \%$ and SF: $5 / 62,8.1 \% ; p=0.057$ ). More patients in the TM group had hospitalization $(15 / 60,25.0 \%$ vs $7 / 62,11.3 \% ; p=0.049)$ within the FU period, which was no longer statistically significant after adjusting for the baseline PGA. The proportions of patients remained in LLDAS were similar in the 2 groups (TM: $75.0 \%$ vs SF: $74.2 \%, p=0.919$ ). None of the patients had COVID-19.

Conclusion: TM resulted in better patient satisfaction and could achieve similar disease control in patients with LN in the short-term when compared to standard care. 
Table 1. Baseline clinical data of the recruited patients and comparison between the telemedicine/standard follow-up groups

\begin{tabular}{lcccc}
\hline & $\begin{array}{c}\text { Overall } \\
(\mathrm{n}=122)\end{array}$ & $\begin{array}{c}\text { Telemedicine } \\
\text { group }(\mathrm{n}=60)\end{array}$ & $\begin{array}{c}\text { Standard follow- } \\
\text { up group }(\mathrm{n}=62)\end{array}$ & P-value \\
\hline Age in years & $44.4 \pm 11.5$ & $44.1 \pm 11.7$ & $44.7 \pm 11.5$ & 0.779 \\
Gender: Female & $111(91.0)$ & $55(91.7)$ & $56(90.3)$ & 0.796 \\
Disease duration in years & $15.1 \pm 9.0$ & $16.2 \pm 8.7$ & $14.0 \pm 9.1$ & 0.115 \\
Nephritis class III, IV or V & $108(88.5)$ & $54(90.0)$ & $54(87.1)$ & 0.427 \\
24 hour urine proteinuria in gram & $0.51 \pm 0.63$ & $0.53 \pm 0.60$ & $0.50 \pm 0.65$ & 0.712 \\
Current use of prednisolone & $112(91.8)$ & $57(95.0)$ & $55(88.7)$ & 0.323 \\
Daily prednisolone dose in mg & $5.51 \pm 4.21$ & $5.69 \pm 4.17$ & $5.34 \pm 4.29$ & 0.570 \\
Use of immunosuppressant & $90(73.8)$ & $46(76.7)$ & $44(71.0)$ & 0.474 \\
SLEDAI-2K & $3.65 \pm 2.33$ & $4.00 \pm 2.34$ & $3.30 \pm 2.29$ & 0.097 \\
PGA & $0.56 \pm 0.65$ & $0.67 \pm 0.69$ & $0.45 \pm 0.60$ & 0.003 \\
LLDAS & $78(63.9)$ & $36(60.0)$ & $42(67.7)$ & 0.251 \\
Remission & $0(0)$ & $0(0)$ & $0(0)$ & $n / a$ \\
Presence of comorbidity & $87(71.3)$ & $40(66.7)$ & $47(75.8)$ & 0.264 \\
SDI & $0.93 \pm 1.15$ & $1.08 \pm 1.28$ & $0.78 \pm 0.98$ & 0.243 \\
HAQ-DI & $0.23 \pm 0.46$ & $0.25 \pm 0.47$ & $0.21 \pm 0.44$ & 0.571 \\
HADS: & $6.07 \pm 4.12$ & $6.20 \pm 4.19$ & $5.93 \pm 4.09$ & 0.720 \\
Anxiety scale & $5.72 \pm 4.31$ & $5.73 \pm 3.93$ & $5.70 \pm 4.68$ & 0.724 \\
Depression scale & & & & \\
\hline
\end{tabular}

Data are reported as mean \pm SD or number (\%). LLDAS: lupus low disease activity state; SDI: Systemic Lupus International Collaborating Clinics/American College of Rheumatology (SLICC/ACR) Damage Index; HAQ-DI: Health Assessment Questionnaire Disability Index; and HADS: Hospital Anxiety and Depression Scale.

A.
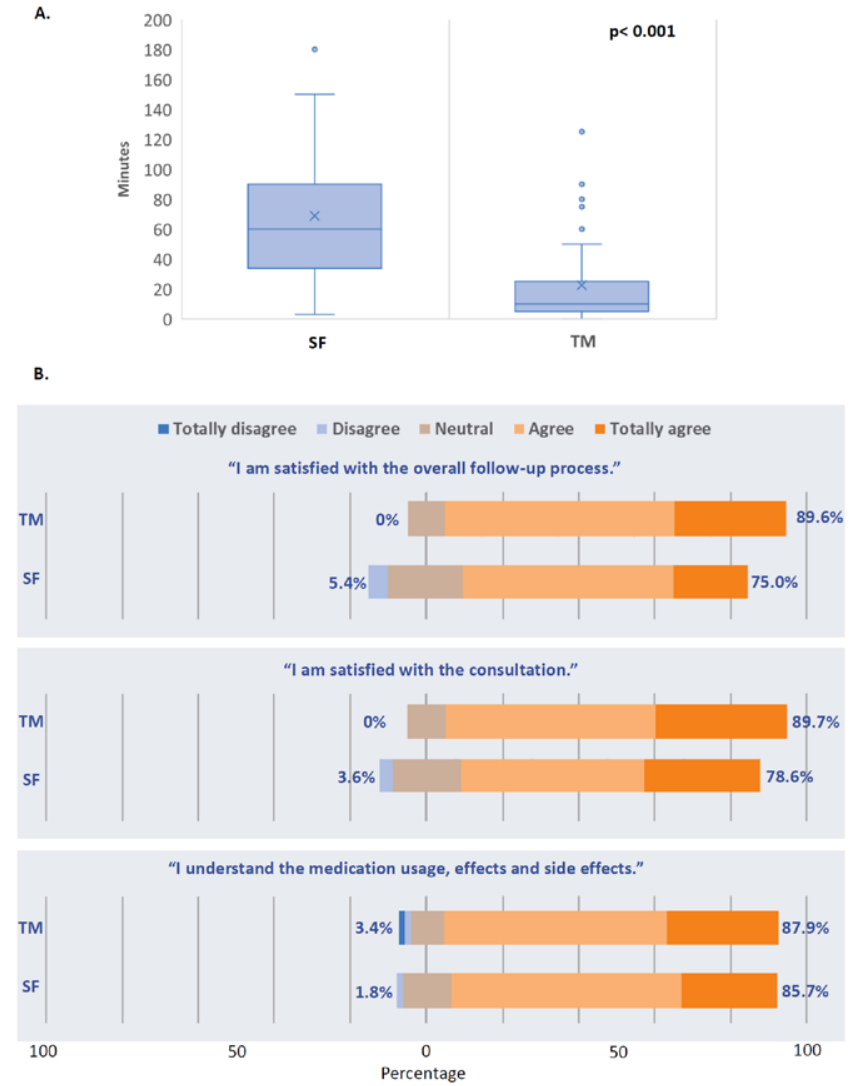

Figure 1A. Mean waiting time between entering the clinic waiting room (virtual or real) and seeing rheumatologist. $\mathrm{TM}=$ Telemedicine, $\mathrm{SF}=$ Standard follow-up. Figure 1B. The results of the postconsultation satisfaction questionnaire. Response is shown as percentage with positive responses on the right. The neutral category was removed when calculating percentages.

Disclosure of Interests: Ho SO: None declared, Evelyn Chow: None declared, Tena K. Li: None declared, Sze-Lok Lau: None declared, Isaac T. Cheng: None declared, Cheuk-Chun Szeto: None declared, Lai-Shan Tam Grant/research support from: Grants from Novartis and Pfizer. DOI: 10.1136/annrheumdis-2021-eular.580

\section{POS1165 LIFESTYLE AND MOOD CHANGES IN AXSPA PATIENTS DURING THE QUARANTINE PERIOD}

E. Erpek ${ }^{1}$, D. Solmaz ${ }^{1}$, E. Durak Ediboglu ${ }^{1}$, G. Alp ${ }^{1}$, E. Otman Akat ${ }^{1}$, H. Cinakli ${ }^{1}$, G. Kabadayi ${ }^{1}$, I. Kurut Aysin ${ }^{1}$, O. Bayindir ${ }^{1}$, S. Gucenmez ${ }^{1}$, M. Ozmen ${ }^{1}$, S. Akar'. ' 'Izmir Katip Celebi University Faculty of Medicine, Rheumatology, Izmir, Turkey
Background: On March 11, 2020, the World Health Organization declared coronavirus disease 2019 (COVID-19) as a pandemic, and mandatory quarantine was applied in Turkey between April and June 2020. With this sanction, sudden changes occurred in a routine lifestyle.

Objectives: This study aimed at evaluating physical activity changes, pres ence of anxiety and depression, altered eating habits and their relationship with disease activity in axial spondyloarthritis (AxSpA) patients during the quarantine period.

Methods: AxSpA patients, who were examined in the rheumatology clinic in the last year before the pandemic period and their relatives were included in this study and were contacted by phone to participate. A structured questionnaire form was performed which included the following data: questions about demographic characteristics, medication use, disease activity scales; BASDAI, BASFI, Patient acceptable symptom state (PASS), patient-reported physical activity state, Short Questionnaire to Assess Health enhancing physical activity (SQUASH), Three-Factor Eating Questionnaire (TFEQ-21), and Hospital Anxiety and Depression Scale (HADs).

Results: 204 AxSpA patients and 106 patients' relatives were contacted in the study (Figure 1). The frequency of male sex and alcohol consumption was higher in the AxSpA compared to the relatives and, other demographic features were summarized in Table $1.30 \%$ of AxSpA patients and $37 \%$ of patient relatives were gained weight with mean $4.5 \pm 2.4$ and $4.4 \pm 3.4$ kilograms, respectively. Weight gain were similar male and female in AxSpA (26.2\% vs $37.2 \%, p>0.05)$. However, the men in the AxSpA group gained more weight than relatives group $(26.2 \%$ vs $7 \%, p<0.05)$. Weight gain group had decreased physical activity than stable group in AxSpA patients (54.8\% vs $37.8 \%, p<0.05)$. We showed mild negative correlation between BASDAI and BASFI scores with SQUASH- total activity score $(r:-0.15, p<0.05$ $r:-0.25, p<0.001$, respectively). Anxiety prevalence were found slightly higher in patients group but not significantly $(40.2 \%$ vs $32.1 \%$; $>0.05)$. Depression were much higher in AxSpA group than relatives $(43.6 \%$ vs $28 \%, p<0.001)$. Depression and anxiety were correlated with disease activity (HADs Depression vs BASDAI r:0.380, $p<0.001$; HADs Anxiety vs BASDAI r:0.418, $\mathrm{p}<0.001$ ) and function (HADs Depression vs BASFI r:0.342, $p<0.001$; HADs Anxiety vs BASFI r:0.313, $p<0.001$ ). Among eating habits uncontrolled and emotional eating scores were showed low correlation with anxiety ( $r: 0.169, p<0.05 ; r: 0.163, p<0.05$, respectively).

Conclusion: One third of our patients were weight gain and approximately half of them had decreased physical activity but we did not show relation between these parameters and disease related factors in the limited period. In addition to that depression and anxiety were detected significant part of AxSpA patients and both of them were correlated with disease activity.

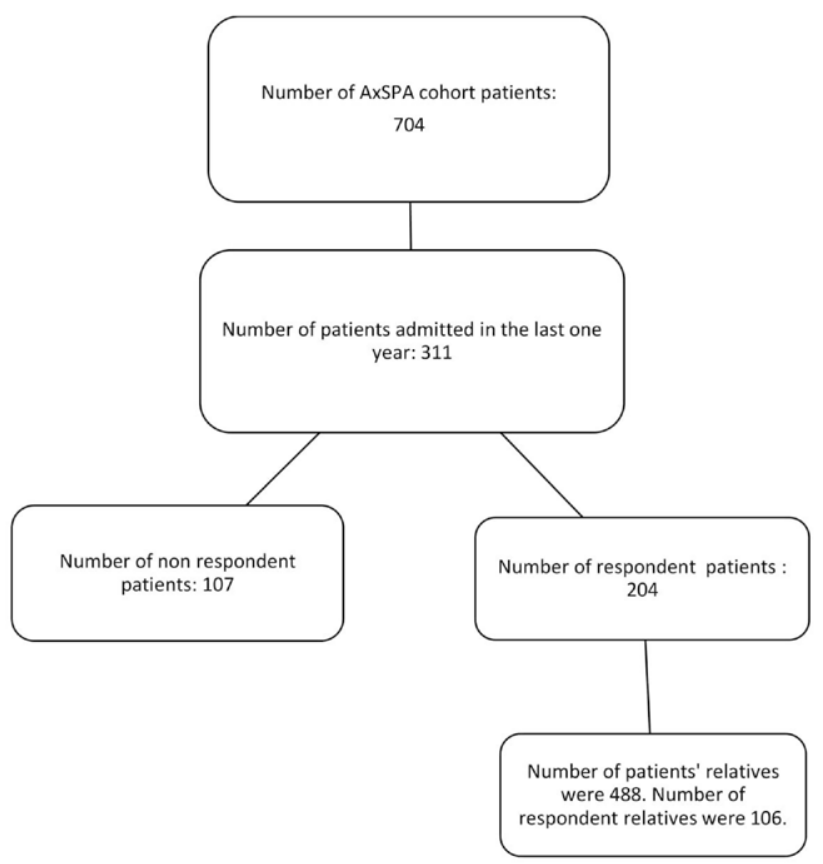

Figure 1: Flow chart of study population 